\title{
A Second Order Sliding Mode Differentiator with a Variable Exponent
}

\author{
M. Ghanes ${ }^{1}$, J.P. Barbot ${ }^{2}$ L. Fridman ${ }^{3}$ and A. Levant ${ }^{4}$
}

\begin{abstract}
In this article, a new second order sliding mode differentiator with a variable exponent is proposed. Inspired by the classical super twisting differentiator, the dedicated differentiator allows to give a solution for reducing the effect of variable noise of sensor output measurement. To achieve this objective, the parameter $\alpha$ that is fixed in a super twisting differentiator is made variable in the proposed differentiator. First of all, the proposed differentiator is presented in free noise case, after that the extension to the case with output noise is given in details. In both cases the practical convergences of the observation error are guaranteed. In the first the radius of the practical stability is depending on the considered unknown input while in the second case this radius depends also on the noise. Finally some simulation results are given in order to show the performances and the effectiveness of the proposed differentiator compared to existing one.
\end{abstract}

\section{INTRODUCTION}

A problem of a real signal derivative estimation is considered in this paper. This problem is well known and has been widely studied in different aspects [2], [5], [8], [9], [11], [12], [13], [14], [18], [19], [20]. With respect to the differentiator design, three main strategies have been considered in the literature. The basic one is based on Euler's differentiator. It is well known that this differentiator doesn't perform well in the presence of a noisy real measured signal. In this case the output of the Euler approximation will be contaminated by the noise and the amplitude of the signal noise's derivative will be amplified. The second strategy is based on numerical differentiator's. The main drawback of this strategy is the phase lag of their filters when the real measured signal is noisy. This article is focused on the third strategy which is based on higher order sliding mode differentiator. This type of differentiator is well investigated in [5] featuring finite-time convergence as an application of the well-known super-twisting algorithm [4]. Particularly the so-called homogeneous differentiator [8], [9] is addressed in this paper. The well-known highgain differentiator [7] and exact high-order sliding mode differentiator [6] can be obtained as particular cases of the homogeneous differentiator, when the homogeneity degree [9] tends respectively to 0 and -1 , respectively [8]. In freenoise case, when the real measurement signal is not affected by the noise, it is well known that the differentiator's can give an exact signal derivative (see for example [4] and [5]). However due to the presence of a variable noise in many real-time applications, which affects the output signal

\footnotetext{
${ }^{1}$ M. Ghanes, IRCCyN, CNRS, Ecole Centrale de Nantes, France Malek. Ghanes@irccyn.ec-nantes.fr

${ }^{2}$ J-P. Barbot, Quartz/ENSEA, 95014 Cergy-Pontoise, France

${ }^{3}$ L. Fridman, UNAM, Mexico

${ }^{4}$ A. Levant, University of Tel Aviv, Israel
}

measurement, the accuracy of the signal derivative provided by the differentiators is degraded. It is well-known that the accuracy of the homogeneous differentiator is linked to the parameter $\alpha$ which is the power of the absolute value of the estimation error. To our best of knowledge the parameter $\alpha$ is always considered fixed in the homogeneous differentiators of the existing literature. This may degrades the real signal derivative estimation in presence of a variable noise affecting the real output (measured) signal. From these considerations, in this paper, under unknown input, a second sliding mode differentiator making the parameter $\alpha$ variable according to the noise is proposed. Note that adaptive schemes of sliding mode controllers exist in the literature ([15], [16], [17], [21], [22], [23], [24], [25], [26], [27]) but to our best of knowledge, there is no results yet in the literature dealing with a variable exponent of higher order sliding mode differentiators schemes. For schemes of differentiators with variables gains, see the works of [2] and [11]. The rest of the paper is organized as follows. Section 2 introduce the system under consideration. In section 3 the proposed differentiator is presented in free noise case but with unknown input and an extension to the case with output noise is given in section 4 . The convergence proves are given in both cases where practical convergences (section 3 and section 4 ), respectively for free noise and output noise, are ensured. In section 5 some simulation results are given in order to show the performances and the effectiveness of the proposed differentiator compared to existing ones when the parameter $\alpha$ is considered to be fixed.

\section{SYSTEM DESCRIPTION}

For simplicity design, in this work one considers the following second order system which refers to a double integrator (in mechanic this corresponds to a simple Lagrangian system) with noisy measurement

$$
\Sigma:\left\{\begin{array}{l}
\dot{x}_{1}=x_{2} \\
\dot{x}_{2}=u \\
y(t)=x_{1 m}=x_{1}+w
\end{array}\right.
$$

where $x(t) \in R^{2}$ is the state of the system, $u(t) \in R$ is the unknown input, $y(t) \in R$ represents the output of the system and $w$ refers to the measurement noise.

\section{Assumptions 1:}

1. The unknown input $u$ is supposed to be bounded, i.e., $|u(t)| \leq u_{\max } \forall t \geq 0$, where $u_{\max }$ is the constant bound value of $u(t)$.

2. The measurement noise $w$ is supposed to be bounded, i.e., $|w(t)| \leq w_{\max } \forall t \geq 0$, where $w_{\max }$ is the constant 
bound value of $w(t)$.

\section{PRoposed DifFERENTIATOR Without MEASUREMENT NOISE}

The proposed differentiator of system (1) is designed as follows:

$$
O:\left\{\begin{array}{l}
\dot{\hat{x}}_{1}=\hat{x}_{2}+k_{1} \mu\left|e_{1}\right|^{\alpha} \operatorname{sign}\left(e_{1}\right) \\
\hat{\hat{x}}_{2}=k_{2} \alpha \mu^{2}\left|e_{1}\right|^{2 \alpha-1} \operatorname{sign}\left(e_{1}\right) \\
\alpha=\frac{\left|e_{1}\right|}{\epsilon_{1}}+1 \\
\left|e_{1}\right|+\epsilon_{2} \\
\hat{y}(t)=\hat{x}_{1} \\
e_{1}=x_{1}-\hat{x}_{1}
\end{array}\right.
$$

where:

- $e_{1}$ is the output observation error. In the same way $e_{2}=$ $x_{2}-\hat{x}_{2}$ is the observation error with respect to unmeasured sub-state.

- $\alpha$ is the variable exponent depending on the observation error $e_{1}$.

For a fixed $\alpha$ and according for example to [10], the degree of homogeneity, $d=\alpha-1$ and the weight $r_{1}$ and $r_{2}$ are equal respectively to 1 and $\alpha$.

\section{A. Observer parameters' definitions and constraints}

- $k_{i}, i=1,2$ are constant positive gains linked to the linear part chosen so that the eigenvalues of the observation error are sufficiently stables and chosen sufficiently large to cancel the effect of the unknown input (see the proof of theorem $1)$.

- $\mu$ is a positive parameter which preserves the homogeneity for a fixed $\alpha$ (see the proof of theorem 1).

- $\epsilon_{1} \in[1,2]$. Hereafter, it is chosen equal to 2 to have $\alpha \in$ $[0.5,1]$ when $e_{1}$ tends to $\infty$.

- $\epsilon_{2}$ will be chosen in the sequel to have $\alpha \in[0.5,1]$ when $e_{1}$ is close to zero.

\section{B. Stability analysis}

Let us consider the observation errors $e_{1}=x_{1}-\hat{x}_{1}$ and $e_{2}=x_{2}-\hat{x}_{2}$ between system (1) and differentiator (2) whose dynamics are given by:

$$
\left\{\begin{array}{l}
\dot{e}_{1}=e_{2}-k_{1} \mu\left|e_{1}\right|^{\alpha} \operatorname{sign}\left(e_{1}\right) \\
\dot{e}_{2}=u-k_{2} \alpha \mu^{2}\left|e_{1}\right|^{2 \alpha-1} \operatorname{sign}\left(e_{1}\right)
\end{array}\right.
$$

Then one establish the following result.

Theorem 1: Under definitions and constraints given in III-A, and assumption 1-1, system (2) is a second sliding mode differentiator of system (1) which, without noisy measurement, converges practically to a ball whose radius depending of $u$.

Proof: Let us take the following change of coordinates

$$
\left\{\begin{array}{l}
\Phi_{1}=\mu\left|e_{1}\right|^{\alpha} \operatorname{sign}\left(e_{1}\right) \\
\Phi_{2}=e_{2}
\end{array}\right.
$$

This change of coordinates is a one to one function, differentiable everywhere expected in $e_{1}=0$.
It is necessary to compute the derivative of $\alpha$ in order to compute the derivative of $\Phi_{1}$ in (4), this derviative is given

$$
\dot{\alpha}=\left(\frac{\frac{1}{\epsilon_{1}} \operatorname{sign}\left(e_{1}\right)}{\left|e_{1}\right|+\epsilon_{2}}-\frac{\left(1+\frac{1}{\epsilon_{1}}\right) \operatorname{sign}\left(e_{1}\right)}{\left(\left|e_{1}\right|+\epsilon_{2}\right)^{2}}\right) \dot{e}_{1}
$$

By replacing $\dot{e}_{1}$ by its expression (3) in (5), the derivative of $\alpha$ becomes

$$
\dot{\alpha}=\frac{\left(0.5 \epsilon_{2}-1\right) \operatorname{sign}\left(e_{1}\right)}{\left(\left|e_{1}\right|+\epsilon_{2}\right)^{2}}\left(e_{2}-k_{1} \mu\left|e_{1}\right|^{\alpha} \operatorname{sign}\left(e_{1}\right)\right)(6)
$$

From (6), it can be seen that the parameter $\epsilon_{2}$ should be chosen as $\epsilon_{2}<2$. Moreover $\epsilon_{2}$ should be greater that 1 , i.e. $\epsilon_{2}>1$, according to our claim that $\alpha$ must be between 0.5 and 1 .

Now, consider the following Lyapunov function candidate

$$
V=\Phi^{T} P \Phi
$$

where $\Phi=\left(\Phi_{1} ; \Phi_{2}\right)$ and $P=P^{T}$ a constant matrix that satisfy

$$
A\left(e_{1}\right)^{T} P+P A\left(e_{1}\right)=-Q\left(e_{1}\right)
$$

where $\mathrm{Q}$ is a definite positive matrix and $A=$ $\left(\begin{array}{cc}-\beta_{1} & 1 \\ -\beta_{2}\left(e_{1}\right) & 0\end{array}\right)$, with $\beta_{1}$ and $\beta_{2}\left(e_{1}\right)$ are given in the next. The notations $A=A\left(e_{1}\right), \beta_{2}=\beta_{2}\left(e_{1}\right)$ and $Q=Q\left(e_{1}\right)$ are chosen to facilitate the reading.

The time derivative of (20) along the trajectories of (3) reads

$$
\dot{V}=\dot{\Phi}^{T} P \Phi+\Phi^{T} P \dot{\Phi}
$$

Derivative of $V(9)$ depends on the derivative of $\Phi$. The letter is computed by using expressions in (4), (3) and variable law of $\alpha$ in (6), that is

$$
\begin{aligned}
& \dot{\Phi}_{1}=\mu\left|e_{1}\right|^{\alpha-1}\left(\alpha+\log \left(\left|e_{1}\right|\right)\left|e_{1}\right| \frac{\left(0.5 \epsilon_{2}-1\right)}{\left(\left|e_{1}\right|+\epsilon_{2}\right)^{2}}\right)\left(e_{2}-k_{1} \Phi_{1}\right) \\
& \dot{\Phi}_{2}=u-k_{2} \alpha \mu^{2}\left|e_{1}\right|^{2 \alpha-1} \operatorname{sign}\left(e_{1}\right)
\end{aligned}
$$

From (10), the derivative of $\mathrm{V}$ in (9) can be rewritten with

$$
\dot{V}=\Gamma \Phi^{T}\left(A^{T} P+P A\right) \Phi+\mathcal{U}^{T} P \Phi+\Phi^{T} P \mathcal{U}
$$

where $\mathcal{U}=\left[\begin{array}{ll}0 & u\end{array}\right]^{T}$,

$$
\Gamma=\alpha \mu\left|e_{1}\right|^{\alpha-1}+\mu \log \left(\left|e_{1}\right|\right)\left|e_{1}\right|^{\alpha} \frac{\left(0.5 \epsilon_{2}-1\right)}{\left(\left|e_{1}\right|+\epsilon_{2}\right)^{2}},
$$

$\beta_{1}=k_{1}$ and

$$
\beta_{2}=\frac{\alpha k_{2} \mu\left|e_{1}\right|^{\alpha-1}}{\Gamma}=\frac{\alpha k_{2}}{\alpha+\log \left(\left|e_{1}\right|\right) \frac{\left(0.5 \epsilon_{2}-1\right)}{\left(\left|e_{1}\right|+\epsilon_{2}\right)^{2}}\left|e_{1}\right|}
$$

Setting $k_{2}=1$. The determinant $(\Delta)$ of the matrix $A$ is equal to $\beta_{2}$. Then, the variation of $\Delta$ is between 0.87 and 1.24 for $\epsilon_{2}=1.001$ as shown in Fig. 1. Note that the largest variation is obtained for $\epsilon_{2}$ close to 1 . When $\epsilon_{2}$ is close to 2 , the variation of $\Delta$ is the smallest one and always close to 1 . 


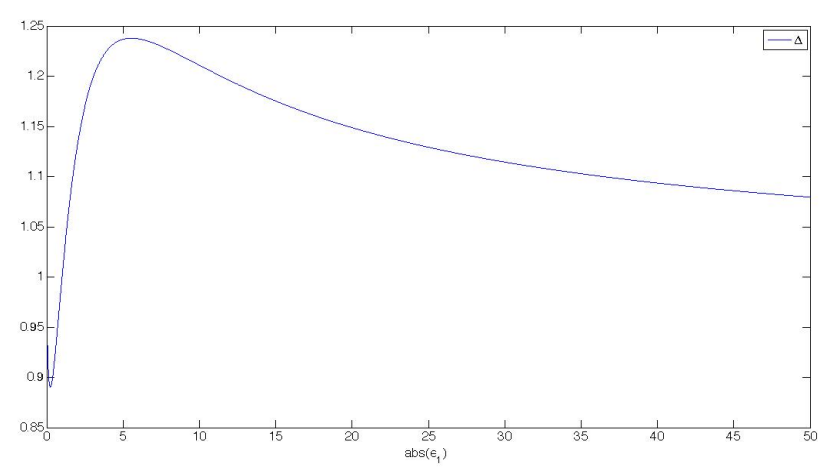

Fig. 1. $\Delta$ versus $\left|e_{1}\right|$ for $\epsilon_{2}=1.001$.

Consequently, the eigenvalues of the matrix $A$ are equal to $\frac{-k_{1} \pm \sqrt{k_{1}^{2}-4 \Delta}}{2}$, which have always a real negative parts. Thus, the derivative of $\mathrm{V}$ in (11), by using (8), becomes

$$
\begin{aligned}
\dot{V} & \leq-\Gamma \Phi^{T} Q \Phi+2 u_{\max } \frac{\|P\| \sqrt{V}}{\sqrt{\lambda_{\min }(P)}} \\
& \leq 0 \quad \forall \sqrt{V} \geq \frac{2 u_{\max }\|P\|}{\Gamma \zeta \sqrt{\lambda_{\min }(P)}}
\end{aligned}
$$

where $\zeta=\lambda_{\min }(Q) / \lambda_{\max }(P)\left(\lambda_{\min }(Q)\right.$ is the smallest eigenvalue of all considered $Q$ ) and $u_{\text {umax }}$ is the maximal value of the unknown input. Even if $\Gamma$ goes to $\infty$ when $e_{1}$ tends to zero for $\alpha \in] 0.51$ ] $e_{2}$ doesn't converge to zero but to a ball whose radius is given by $k_{1} \mu\left(\frac{u_{\max }}{k_{2} \alpha \mu^{2}}\right)^{\frac{\alpha}{2 \alpha-1}}$. Then a practical convergence of the observation error is ensured.

\section{Remarks 1:}

1. The difference of the proof described before and the proof in [1] is the fact that linear matrix $A$ is not directly assigned by the linear parameters $k_{1}$ and $k_{2}$. This is due to the fact that the variable parameter $\alpha$ modify both parts, the linear one (A) and the homogeneity gain $\Gamma$ given by (12) in the derivative of $\mathrm{V}(21)$.

2. Note that when $\alpha=0.5$, the finite time convergence of the differentiator error is obtained because in this case the perturbation due to $u$ is directly canceled by the sign function for sufficiently large $\mu$. Note that in this case the radius of the ball convergence cannot be defined by $k_{1} \mu\left(\frac{u_{\max }}{k_{2} \alpha \mu^{2}}\right)^{\frac{\alpha}{2 \alpha-1}}$.

\section{PROPOSED DIFFERENTIATOR WITH MEASUREMENT NOISE}

In the previous section, it was shown that it is possible to modify the parameter $\alpha$ with respect to the output observation error (in the case of free noise). Hereafter, the considered problem is to propose the same type of differentiator with measurement noise. In order to avoid instantaneous variations of parameter $\alpha$ due to noisy output measurement, it is introduced a low pass-filter between the output measurement observation error and $\alpha$. Consequently the parameter $\alpha$ is only function of the state observation error $e_{1}$ and the expected absolute value of noise $w$. It is important to mention that only the parameter $\alpha$ is filtered and by doing this the delay due to the filter is avoided in the observation state.

$$
O:\left\{\begin{array}{l}
\dot{\hat{x}}_{1}=\hat{x}_{2}+k_{1} \mu\left|e_{1 m}\right|^{\alpha} \operatorname{sign}\left(e_{1 m}\right) \\
\dot{\hat{x}}_{2}=k_{2} \alpha \mu^{2}\left|e_{1 m}\right|^{2 \alpha-1} \operatorname{sign}\left(e_{1 m}\right) \\
\dot{x}_{3}=-\frac{x_{3}}{\tau}+\left|e_{1 m}\right| \\
\alpha=\frac{\left|x_{3}\right|}{\epsilon_{1}}+1 \\
\left|x_{3}\right|+\epsilon_{2} \\
\hat{y}(t)=\hat{x}_{1} \\
e_{1 m}=x_{1 m}-\hat{x}_{1}
\end{array}\right.
$$

where:

- $e_{1 m}$ is the output observation error. Contrarily to $e_{1}$ which is the observation error whith respect to the first state component.

- $x_{3}$ is a sufficiently filtered output observation error of $\left|e_{1 m}\right|$, in order to be able to consider that its derivative is equal to zero in fast dynamics (see the proof of Theorem 2).

- $\alpha$ is the variable exponent depending on the filtered output observation error $x_{3}$ in order to be variable with respect to the noise $w$.

For a fixed $\alpha$ and according for example to [10] , the degree of homogeneity, $d=\alpha-1$ and the weight $r_{1}$ and $r_{2}$ are equal respectively to 1 and $\alpha$. In this case, as $\alpha$ is slowly variable, the assumption of a fixed $\alpha$ is closely satisfied.

\section{A. Observer parameters' definitions and constraints}

- $k_{i}, i=1,2$ are constant positive gains linked to the linear part chosen so that the eigenvalues of the observation error are sufficiently stables and chosen sufficiently large to cancel the effect of the unknown input (see the proof of Theorem 2).

- $\mu$ is a positive parameter which preserves the homogeneity (see the proof of theorem 2).

- $\epsilon_{1}$ is fixed to be 2 to have $\alpha \in[0.5,1]$ when $e_{1}$ tends to $\infty$.

- $\epsilon_{2}$ will be chosen in the sequel to have $\alpha \in[0.5,1]$ when $e_{1}$ is close to zero.

- $\tau$ is a positive constant parameter that will be chosen in order to fix the frequency range of the filter $x_{3}$ sufficiently low.

Let us consider the same observation errors as in previous case (free noise), that is $e_{1}=x_{1}-\hat{x}_{1}$ and $e_{2}=x_{2}-\hat{x}_{2}$ between system (1) and the second differentiator (15) whose dynamics are given by:

$$
\left\{\begin{array}{l}
\dot{e}_{1}=e_{2}-k_{1} \mu\left|e_{1 m}\right|^{\alpha} \operatorname{sign}\left(e_{1 m}\right) \\
\dot{e}_{2}=u-k_{2} \alpha \mu^{2}\left|e_{1 m}\right|^{2 \alpha-1} \operatorname{sign}\left(e_{1 m}\right)
\end{array}\right.
$$

The folowing result is established.

Theorem 2: Under definitions-constraints given in IVA and assumptions 1-1 and 1-2, system (15) is a second order sliding mode differentiator with a variable exponent of system (1) with noisy measurement, which converges to a ball whose radius is function of $|w|$ and $|u|$.

Proof: Let us take the following change of coordinates

$$
\left\{\begin{array}{l}
\Phi_{1}=\mu\left|e_{1}\right|^{\alpha} \operatorname{sign}\left(e_{1}\right) \\
\Phi_{2}=e_{2}
\end{array}\right.
$$


The derivative of $\alpha$ which is necessary in the next, is given by

$$
\dot{\alpha}=\left(\frac{\frac{1}{\epsilon_{1}} \operatorname{sign}\left(x_{3}\right)}{\left|x_{3}\right|+\epsilon_{2}}-\frac{\left(1+\frac{1}{\epsilon_{1}}\right) \operatorname{sign}\left(x_{3}\right)}{\left(\left|x_{3}\right|+\epsilon_{2}\right)^{2}}\right) \dot{x}_{3}
$$

This derivative (18) can be simplified as follows

$$
\dot{\alpha}=\frac{\left(0.5 \epsilon_{2}-1\right) \operatorname{sign}\left(x_{3}\right)}{\left(\left|x_{3}\right|+\epsilon_{2}\right)^{2}} \dot{x}_{3}
$$

From (19), it can be seen that the parameter $\epsilon_{2}$ should be chosen as $\epsilon_{2}<2$. Moreover $\epsilon_{2}$ should be greater that 1 , i.e. $\epsilon_{2}>1$, according to our claim that $\alpha$ must be between 0.5 and 1 .

Now, consider the following Lyapunov function candidate

$$
V=\Phi^{T} P \Phi
$$

where $\Phi=\left(\Phi_{1} ; \Phi_{2}\right)$ and $P=P^{T}$ a positive constant matrix.

The time derivative of (20) along the trajectories of (16) reads

$$
\dot{V}=\dot{\Phi}^{T} P \Phi+\Phi^{T} P \dot{\Phi}
$$

Derivative of $\mathrm{V}$ (21) depends on the derivative of $\Phi$. The letter is computed by using expressions in (4), (16) and variable law of $\alpha$ in (6), that is

$$
\begin{aligned}
\dot{\Phi}_{1}= & \mu \alpha\left|e_{1}\right|^{\alpha-1}\left(\Phi_{2}-k_{1} \mu\left|e_{1 m}\right|^{\alpha} \operatorname{sign}\left(e_{1 m}\right)\right) \\
& +\mu \log \left(\left|e_{1}\right|\right)\left|e_{1}\right|^{\alpha} \operatorname{sign}\left(e_{1}\right) \frac{\left(0.5 \epsilon_{2}-1\right) \operatorname{sign}\left(x_{3}\right)}{\left(\left|x_{3}\right|+\epsilon_{2}\right)^{2}} \dot{x}_{3} \\
\dot{\Phi}_{2}= & u-k_{2} \alpha \mu^{2}\left|e_{1 m}\right|^{2 \alpha-1} \operatorname{sign}\left(e_{1 m}\right)
\end{aligned}
$$

By invoking the singular perturbation theorem (see [3]), $\dot{x}_{3}$ in (22) can be considered equal to zero due to the requirement on the low-pass filter (see subsection IV-A). Nevertheless, without invoking singular perturbation theorem, in the first equation of (22), it can be seen that when $e_{1}$ goes to zero then $\dot{x}_{3}$ is not multiplied by $\infty$ contrarily to the first term of $\dot{\Phi}_{1}$. So, it is possible to rewrite (22) as follows

$$
\begin{aligned}
& \dot{\Phi}_{1}=\mu \alpha\left|e_{1}\right|^{\alpha-1}\left(\Phi_{2}-k_{1} \mu\left|e_{1 m}\right|^{\alpha} \operatorname{sign}\left(e_{1 m}\right)\right) \\
& \dot{\Phi}_{2}=u-k_{2} \alpha \mu^{2}\left|e_{1 m}\right|^{2 \alpha-1} \operatorname{sign}\left(e_{1 m}\right)
\end{aligned}
$$

From the following inequalities, it is obtained:

- For $e_{1}+w \geq 0$ with $\left\lfloor e_{1 m}\right\rceil^{\alpha}=\left|e_{1}+w\right|^{\alpha} \operatorname{sign}\left(e_{1}+w\right)$ :

$\left|e_{1}\right|^{\alpha} \operatorname{sign}\left(e_{1}\right)-2|w|^{\alpha} \leq\left\lfloor e_{1 m}\right\rceil^{\alpha} \leq\left|e_{1}\right|^{\alpha} \operatorname{sign}\left(e_{1}\right)+2|w|^{\alpha}$

- For $e_{1}+w \leq 0$ :

$\left|e_{1}\right|^{\alpha} \operatorname{sign}\left(e_{1}\right)+2|w|^{\alpha} \geq\left\lfloor e_{1 m}\right\rceil^{\alpha} \geq\left|e_{1}\right|^{\alpha} \operatorname{sign}\left(e_{1}\right)-2|w|^{\alpha}$

Then from previous equations, there exists a function $f\left(e_{1}, w\right) \in[-2,2]$ such that

$$
\left\lfloor e_{1 m}\right\rceil^{\alpha}=\left|e_{1}\right|^{\alpha} \operatorname{sign}\left(e_{1}\right)+f\left(e_{1}, w\right)|w|^{\alpha} .
$$

Consequently, expression (23) becomes

$$
\begin{aligned}
\dot{\Phi}_{1}= & \mu \alpha\left|e_{1}\right|^{\alpha-1}\left(\Phi_{2}-k_{1} \mu\left|e_{1}\right|^{\alpha} \operatorname{sign}\left(e_{1}\right)\right. \\
& \left.-k_{1} \mu f_{1}\left(e_{1}, w\right)|w|^{\alpha}\right) \\
\dot{\Phi}_{2}= & u-k_{2} \mu^{2}\left(\left|e_{1}\right|^{2 \alpha-1} \operatorname{sign}\left(e_{1}\right)-f_{2}\left(e_{1}, w\right)|w|^{2 \alpha-1}\right)
\end{aligned}
$$

So one obtains

$$
\begin{aligned}
\dot{\Phi}_{1} & \left.=\alpha \mu\left|e_{1}\right|^{\alpha-1}\left(\Phi_{2}-k_{1} \Phi_{1}\right)+k_{1} \mu f_{1}\left(e_{1}, w\right)|w|^{\alpha}\right) \\
\dot{\Phi}_{2} & =u \\
& -\alpha \mu\left|e_{1}\right|^{\alpha-1}\left(k_{2} \Phi_{1}-k_{2} \mu\left|e_{1}\right|^{1-\alpha} f_{2}\left(e_{1}, w\right)|w|^{2 \alpha-1}\right)
\end{aligned}
$$

Then, by writting (27) in compact form it gives

$$
\dot{\Phi}=\Lambda(A \Phi+N)+\mathcal{U}
$$

where

$$
\begin{array}{r}
N=\left[\begin{array}{c}
k_{1} \mu f_{1}\left(e_{1}, w\right)|w|^{\alpha} \\
k_{2} \mu\left|e_{1}\right|^{1-\alpha} f_{2}\left(e_{1}, w\right)|w|^{2 \alpha-1}
\end{array}\right], \\
A=\left(\begin{array}{ll}
-k_{1} & 1 \\
-k_{2} & 0
\end{array}\right),
\end{array}
$$

and $\Lambda=\alpha \mu\left|e_{1}\right|^{\alpha-1}$. Furthermore, the matrix $P$ is chosen to satisfy the following equality

$$
A^{T} P+P A=-Q
$$

where $Q$ is a constant definite positive matrix. Note that the eigenvalues of matrix $A$ are equal to $\frac{-k_{1} \pm \sqrt{k_{1}^{2}-4 k_{2}}}{2}$ which have always a real negative parts for appropriate choice of $k_{1}$ and $k_{2}$.

Now by replacing (28) in (21) and using (29), the derivative of $\mathrm{V}$ reads

$$
\begin{aligned}
\dot{V} \leq & -\Lambda \Phi^{T} Q \Phi+2 \Lambda N^{T} P \Phi+\mathcal{U}^{T} P \Phi+\Phi^{T} P \mathcal{U} \\
\leq & -\delta \Lambda\|\Phi\|_{P}^{2}+2 \Lambda \lambda_{\max }(P)\|\Phi\|_{P}\|N\| \\
& +2 u_{\max } \frac{\|P\|\|\Phi\|_{P}}{\sqrt{\lambda_{\min }(P)}}
\end{aligned}
$$

where $\delta=\frac{\lambda_{\min }(Q)}{\lambda_{\max }(P)}$ and $\|\Phi\|$ should verify the following inequality

$$
\|\Phi\|_{P} \geq \frac{2 \lambda_{\max }(P)}{\delta}\|N\|_{\max }+\frac{2\|P\|}{\delta \Lambda \sqrt{\lambda_{\max }(P)}} u_{\max }
$$

As long as $\|\Phi\|$ verifies (31), $\dot{V}$ (30) is negative, then this ensures that the error with respect to $\|\Phi\|$ converges to a ball whose radius equal or smaller to $\frac{2 \lambda_{\max }(P)}{\delta}\|N\|_{\max }+$ $\frac{2\|P\|}{\delta \Lambda \sqrt{\lambda_{\max }(P)}} u_{\max }$, consequently $\left|e_{2}\right|$ is smaller or equal than this radius when $t$ tends to $\infty$.

\section{Simulation Results}

In this section, only the differentiator simulation results of section 4 with noise measurement, is presented. Matlab Simulink software is used. The results when the parameter $\alpha$ is fixed are also given to compare the performances in both cases, when the parameter $\alpha$ is variable (our proposed differentiator) and when it is fixed. The noise $w$ shown in Fig. 2-a), is added to the output measurement, i.e., $y=e_{1 m}=e_{1}+w$, to simulate a real situation. The simulated differentiator of system (1) is given by system (15). Note that the signal $u=\sin (0.05 t)$ is considered as an input for system (15) while this signal is considered null by differentiator (1) (unknown input). With respect to 
subsection (IV-A), the parameters' differentiator are chosen as follows $\epsilon_{1}=2, \epsilon_{2}=1.001, k_{1}=10, k_{2}=30, \mu=1$ and $\tau=3$. The filter parameter $\tau$ is fixed to satisfy the singular perturbation aspect enhanced in the proof of Theorem 2 .

The obtained results are shown in Figures 2, 3, 4, 5, 6, 7, 8,9 . Figures $3,4,5,6$ show the results of the second state $x_{2}$ and its observation $\hat{x}_{2}$ when $\alpha$ is variable and when it is fixed, while in Figures 7, 8, 9, the observation errors are displayed. Fig. 2-c) shows the filter response $x_{3}$ when it can be seen that its response increases when the response of the variable parameter $\alpha$ (Fig. 2-b) decreases and vice-versa.

The result of the second state observation $\hat{x}_{2}$ of our proposed differentiator (Fig. 3) shows a better result than all the observation results obtained by fixing all time $\alpha$ to 0.5 (Fig. 4), to 0.7 (Fig. 5) and to 0.9 (Fig. 6). This claim is confirmed by the plotted observation errors of the second state (Figures 7, 8, 9). In each figure, the observation error of our proposed differentiator ( $\alpha$ is variable) is compared to the error when $\alpha$ is fixed. It can be noted that when the amplitude of noise is small and big (see the noise Fig. 2-a)), these errors are well reduced in case of our proposed differentiator because the letter takes a better choice of parameter $\alpha$ (Fig. 2-b)) in both situations (small and big noise amplitude), which means that the error of the second state observation error $\Phi_{2}=e_{2}=x_{2}-\hat{x}_{2}$ goes to a ball whose radius is better reduced than the noted cases (i.e. $\alpha$ is equal to 0.5 or 0.7 or 0.9 ).

a)

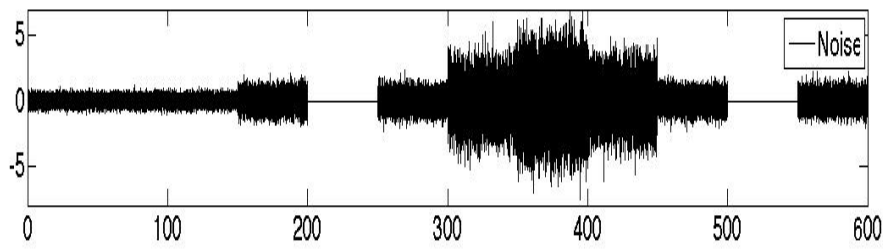

b)

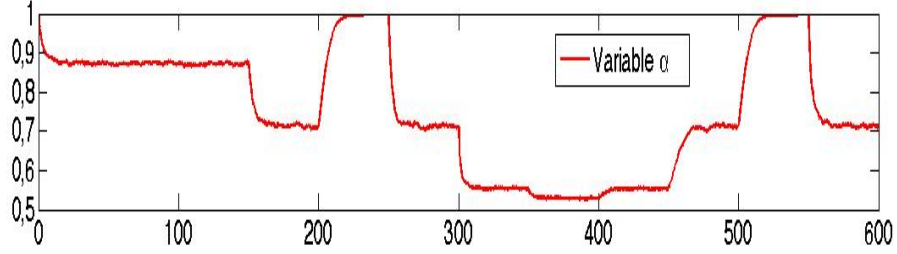

c)

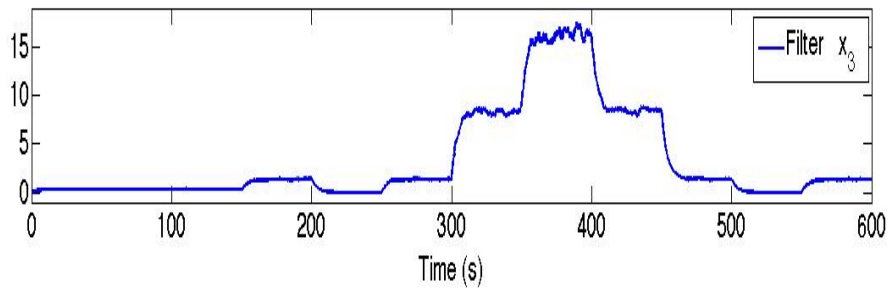

Fig. 2. Noise, Variable $\alpha$, Filter $x_{3}$

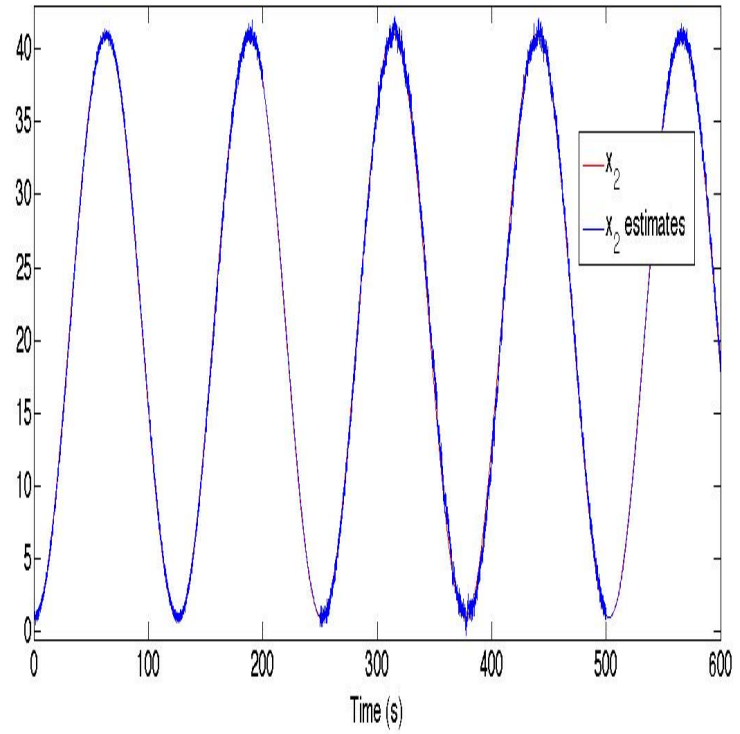

Fig. 3. $x_{2}$ in red and $\hat{x}_{2}$ in blue with variable $\alpha$.

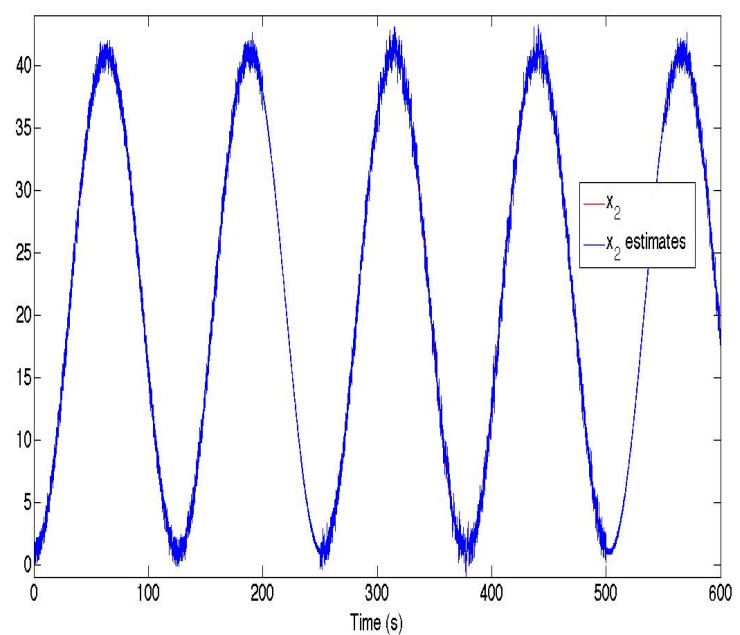

Fig. 4. $x_{2}$ in red and $\hat{x}_{2}$ in blue with $\alpha=0.5$.

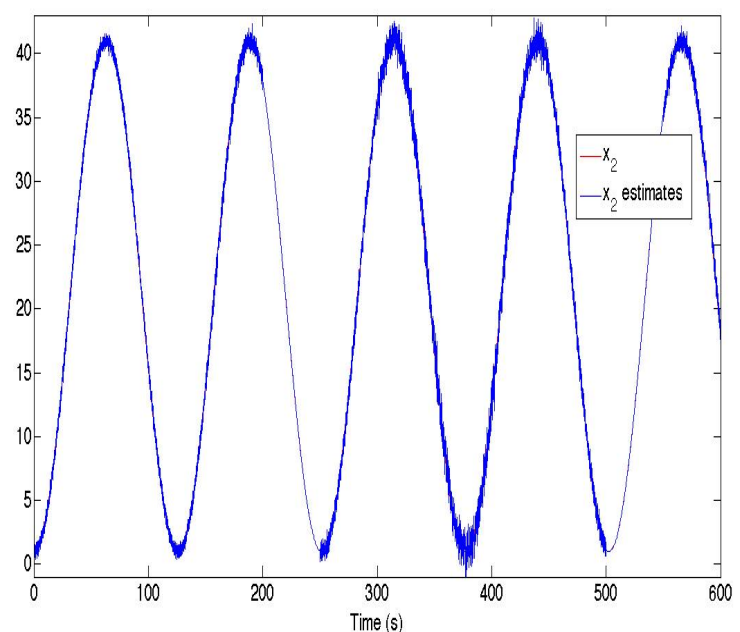

Fig. 5. $\quad x_{2}$ in red and $\hat{x}_{2}$ in blue with $\alpha=0.7$. 


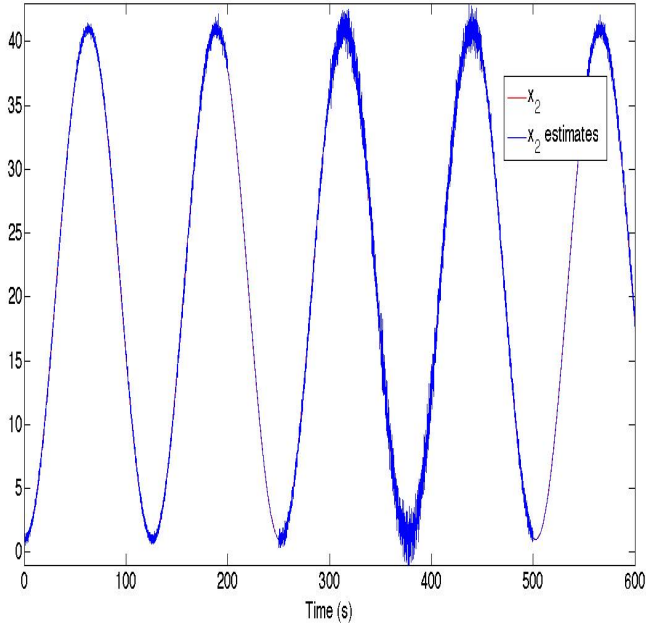

Fig. 6. $x_{2}$ in red and $\hat{x}_{2}$ in blue with $\alpha=0.9$.

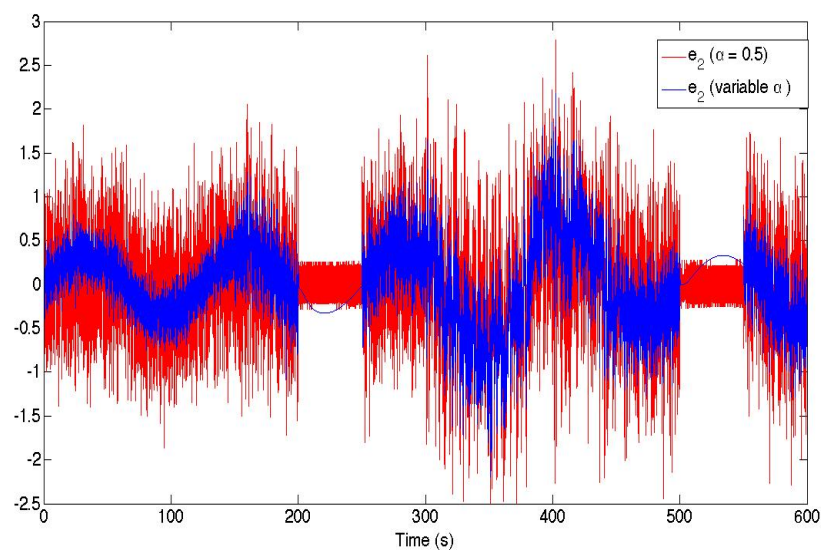

Fig. 7. Error $e_{2}=x_{2}-\hat{x}_{2}$ in blue with variable $\alpha$ and in red with $\alpha=0.5$.

It can be noted that the average value of the error is equal to zero in case of $\alpha=0.5$ without noise measurement and under a unknown input (between $200 \mathrm{~s}$ and $250 \mathrm{~s}$ for example in Fig. 7).

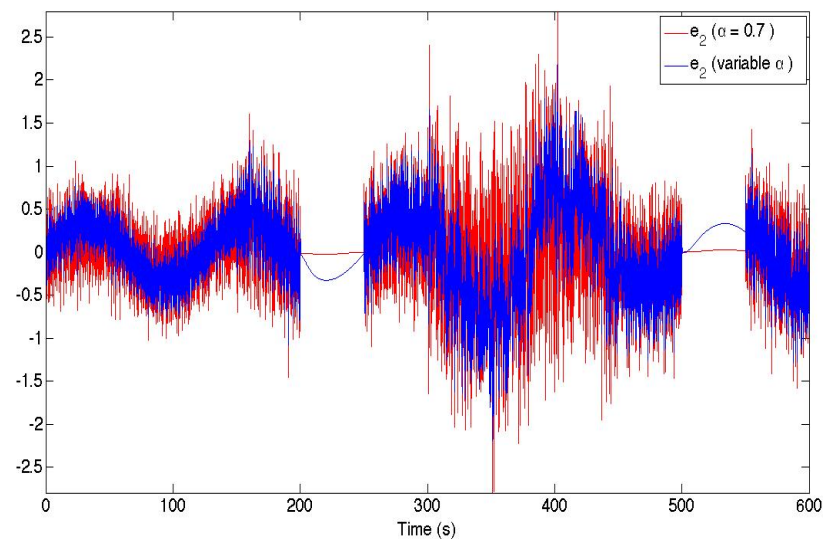

Fig. 8. Error $e_{2}=x_{2}-\hat{x}_{2}$ in blue with variable $\alpha$ and in red with $\alpha=0.7$.

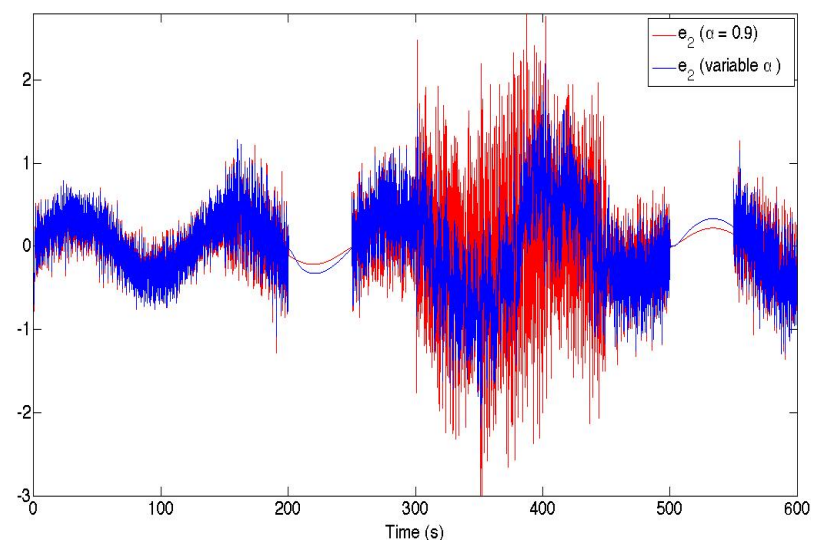

Fig. 9. Error $e_{2}=x_{2}-\hat{x}_{2}$ in blue with variable $\alpha$ and in red with $\alpha=0.9$.

\section{CONCLUSION}

In this paper two differentiators are proposed. Both are based on classical homogeneous differentiator structure where it is added a specific variation of the exponent term. Simulations highlight the well-founded of the dedicated differentiators. Future works will be done on highest order of sliding mode differentiators design and more efficient variation law of $\alpha$.

\section{REFERENCES}

[1] J. Moreno, “ Lyapunov Approach for Analysis and Design of Second Order Sliding Mode Algorithms, " Chapt. 4, Sliding Modes, LNCIS 412, pp. 113-149. Springer-Verlag Berlin Heidelberg 2011.

[2] M.T. Angulo, J. A. Moreno and L. Fridman, “, The differentiation error of noisy signals using the Generalized Super-Twisting differentiator " IEEE Conference on Decision and Control, December 10-13, Maui, Hawaii, USA, 2012.

[3] A.N. Tikhonov, A.B. Vasil"eva and V.M. Volosov, Ordinary differential equations. In E. Roubine, editor, Mathematics Applied to Physics, pp 162-228, Springer-Verlag New York, 1970.

[4] A. Levant, "Sliding order and sliding accuracy in sliding mode control,” Int. J. Control, vol. 58, pp. 1247-1263, 1993.

[5] A. Levant, "Robust exact differentiation via sliding mode technique," Automatica, vol. 34, no. 3, pp. 379-384, 1998.

[6] "Higher-order sliding modes, differentiation and output- feedback control," International Journal of Control, vol. 76 (9/10), pp. 924-941, 2003.

[7] K. Khalil, "High-gain observers in nonlinear feedback control," Lecture Notes in Control and Information Sciences, vol. 244, pp. 249268, 1999.

[8] A. Polyakov, D. Efimov, W. Perruquetti, Homogeneous Differentiator Design using Implicit Lyapunov Function Method, European Control Conference (ECC), 2014.

[9] W. Perruquetti, T. Floquet, and E. Moulay, "Finite-time observers: Application to secure communication, "IEEE Transactions on Automatic Control, vol. 53(1), pp. 356-360, 2008.

[10] W. Perruquetti and T. Floquet, "Homogeneous nite time observer for nonlinear systems with linearizable error dynamics, "IEEE Conference on Decision and Control, New Orleans, USA, 2007

[11] J. H. Ahrens and H. K. Khalil, "High-Gain Observers in the Presence of Measurement Noise: A Switched-Gain Approach, "17 Th IFAC World congress, Seoul 2008.

[12] H. K. Khalil and L. Praly, "High-gain observers in nonlinear feedback control, “ Int. J. Robust. Nonlinear Control, 2013.

[13] M. Mboup and C. Join, and M. Fliess, "Numerical differentiation with annihilators in noisy environment, " Numerical Algorithms, Vol. 50, no. 4, pp. 439-467, 2009. 
[14] S. Diop and J.W. Grizzle and F. Chaplais, "On Numerical Differentiation Algorithms for Nonlinear Estimation, "IEEE Conference on Decision and Control, Sydney, Australia, 2000.

[15] Ch. Edwards, Y. Shtessel, " Adaptive Continuous Higher Order Sliding Mode Control, “ 19 Th IFAC World congress, South Africa 2014.

[16] Y. Shtessel, M. Taleb, F. Plestan, " A novel adaptive-gain supertwisting sliding mode controller: Methodology and application, “ Automatica, Vol. 48, no. 5, pp. 759-769, May 2012.

[17] F. Plestan, Y. Shtessel, V. Bregeault and A. Poznyak, “ New methodologies for adaptive sliding mode control, " International Journal of Control, Vol. 83, no. 9, pp. 1907-1919, 2010.

[18] H. Hammouri, B. Targui and F. Armanet, "High gain observer based on a triangular structure, " International Journal of Robust and Nonlinear Control, Vol. 12, no. 6, pp. 497-518, 2002.

[19] W. Kang, " Moving horizon numerical observers of nonlinear control systems, " Automatic Control, IEEE Transactions on, Vol. 51, no. 2, pp. 344-350, 2006.

[20] G. Besançon, " High-gain observation with disturbance attenuation and application to robust fault detection, " Automatica Vol. 39, no. 6, pp. 1095-1102, 2003.

[21] V.I. Utkin, and A.S. Poznyak, " Adaptive sliding mode control with application to super-twist algorithm: Equivalent control method, " Automatica Vol. 49, no. 1, 39-47, 2013.

[22] G. Bartolini, A. Ferrara and E. Usai, "Chattering avoidance by secondorder sliding mode control, "IEEE Transactions on Automatic Control, Vol. 43, no. 2, pp. 241-246, 1998.

[23] A. Pisano, M. Tanelli and A. Ferrara, " Combined switched/time-based adaptation in second order sliding mode control, " IEEE Conference on Decision and Control, Florence, Italy, 2013.

[24] G. Bartolini, A., Levant, F., Plestan, M., Taleb and E. Punta, “ Adaptation of sliding modes, "IMA Journal of Mathematical Control and Information, "Vol. 30, no. 3, pp. 285-300, 2013.

[25] B. Mirkin, P.-O. Gutman and Y. Shtessel, " Asymptotic sliding mode control approach to adaptive distributed tracking problem for multiagent nonlinear delayed systems, " International Journal of Control, Vol. 85, no. 11, pp. 1671-1682, 2012.

[26] J. Anet, N. Anelone, Y. Orlov and S. K. Spurgeon, “ Modelling the self-tolerance mechanisms of T cells: An adaptive sliding mode control approach, " UKACC International Conference on Control, pp. 573$578,2014$.

[27] M.L. Hammadih, K. Al Hosani and I. Boiko, "Interpolating sliding mode observer for a ball and beam system, " International Journal of Control Vol. 89, no. 9, 2016. 